\title{
Phason mode in inclusion compounds
}

\author{
L. A. Brussaard, A. Fasolino, and T. Janssen \\ Institute of Theoretical Physics, University of Nijmegen, Postbus 9010, 6500 GL Nijmegen, The Netherlands
}

(Received 16 October 2000; published 15 May 2001)

\begin{abstract}
The phason mode in inclusion compounds is investigated using the double chain model, which consists of two mutually interacting chains. This model generalizes the Frenkel-Kontorova model to a system where both sublattices are deformable. The phase diagram in parameter space contains a region with a zero-frequency phason and a region with a phason gap. In comparison with earlier work, the phase-transition line is not convex. The dynamic structure factor around Bragg and satellite peaks is calculated to find conditions needed to measure the phason mode in experiment.
\end{abstract}

DOI: 10.1103/PhysRevB.63.214302

PACS number(s): 61.44.Fw, 63.20.-e

\section{INTRODUCTION}

Incommensurate composites belong to the class of quasiperiodic crystals. They consist of at least two interpenetrating crystalline subsystems. In one or more crystallographic directions the periodicities of these subsystems are incommensurate, i.e., the ratio of the periodicities is not a rational number.

Examples of such a system are the inclusion compound $\mathrm{C}_{n} \mathrm{H}_{2 n+2}$ /urea for some values of $n$. The urea molecules (the hosts) form a hexagonal lattice, with the alkane molecules (the guests) situated in the channels of this lattice. The ratio of the periodicities of the two subsystems along these channels depends on $n$, or in other words, depends on which alkane is present. For some alkanes this ratio is incommensurate, e.g., heptadecane $(n=17)$. Theoretically these incommensurate systems show the existence of a sliding or phason mode for not too strong interchain coupling. However, experiments are still not conclusive on the existence of this mode ${ }^{1-3}$ in alkane/urea systems.

This paper studies the theoretical aspects of the statics and dynamics of inclusion compounds to find conditions under which the phason mode can be observed in experiment. Earlier work used the Frenkel-Kontorova model, ${ }^{4,5}$ which consists of a harmonic chain of atoms under the influence of a rigid substrate potential. This system describes composites where one of the subsystems is infinitely rigid. Double Frenkel-Kontorova models ${ }^{6,7}$ allowed some understanding of solitons involving deformation of both subsystems. However, these models are not able to explain correctly static properties like the intermodulation or give a full account of the dynamics of both subsystems.

The recently introduced double chain model, ${ }^{8}$ provides a suitable model to investigate inclusion composites as it takes into account the discreteness of the real crystal and the mutual interaction of both sublattices. The model generalizes the Frenkel-Kontorova model, replacing the rigid potential by a second deformable chain. Because both sublattices are now mutually deformable, the influence of the chains on each other can be studied more realistically. A study of friction $^{9}$ also used a double chain model, but here one of the chains was attached to a substrate. Therefore, there is no acoustic mode present in that model.

This paper studies the double chain model starting from its potential energy. Minimizing the energy leads to the ground state of the system. Modulation functions provide information about these ground states. This leads to a phase diagram containing a region in which the modulation functions are analytic and a region in which they are nonanalytic. The dynamical matrix gives the phonon spectrum of the crystal. For weak interchain coupling, two zero-frequency modes exist. One of them is the phason mode, the other one is the acoustic mode. If the interchain potential increases beyond a threshold value the degeneracy in the $\Gamma$ point no longer exists and a phason gap appears. This also leads to a phase diagram, which coincides with the diagram obtained by considering the modulation functions. Finally, the geometrical and dynamical structure factors give insight in the experimental effects of the phason and acoustic modes.

An interchain interaction with a long tail will be introduced in the double chain model. This interaction will lead to an asymmetric transition line in the phase diagram, which differs from the results found earlier using a Gaussian potential. The shape of the transition line is found to depend on the ratio of the numbers of particles in the two chains. The conditions needed to find a phason mode in neutron-scattering experiments are discussed as well.

The paper is organized as follows. Section II presents the model. Section III describes the statics and dynamics of the double chain model, which will lead to the phase diagram. The following section discusses the observation of the phason mode. Section V draws conclusions.

\section{DOUBLE CHAIN MODEL}

The double chain model consists of two parallel chains of particles. Figure 1 visualizes this system. The interaction between particles in one chain (the intrachain interaction) is harmonic. The interaction between particles situated in different chains (the interchain interaction) is Lennard-Joneslike. In a previous work, ${ }^{8}$ a Gaussian potential was used instead of the Lennard-Jones potential. Results for these different potentials will be compared at the end of the next section. The potential energy of the model is written as:

$$
\begin{aligned}
E= & \frac{k_{1}}{2} \sum_{i}\left(x_{i}-x_{i-1}-\alpha\right)^{2}+\frac{k_{2}}{2} \sum_{j}\left(y_{j}-y_{j-1}-\beta\right)^{2} \\
& +\sum_{i} \sum_{j} \Phi\left(x_{i}-y_{j}\right),
\end{aligned}
$$




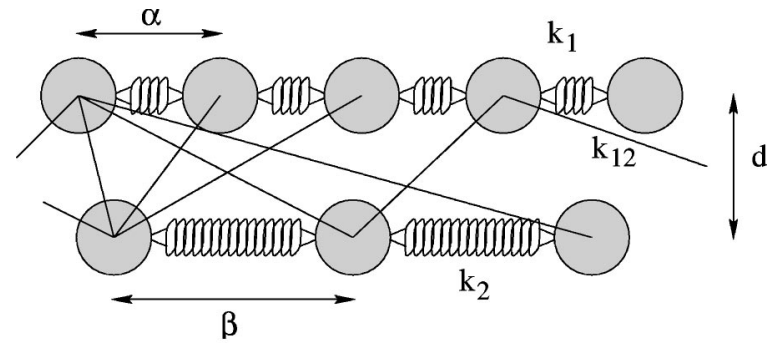

FIG. 1. The double chain model. The intrachain potentials (indicated by springs) are harmonic. The interchain potential is either Lennard-Jones-like or Gaussian. The distance $d$ between the chains is fixed. The equilibrium distances between particles in chains 1 and 2 are $\alpha$ and $\beta$, respectively.

where $k_{1}$ and $k_{2}$ are the force constants of the intrachain potentials, $\alpha$ and $\beta$ are the equilibrium distances, and $x_{i}$ and $y_{j}$ are the positions of the $i$ th $(j$ th) particle of the first and second chain respectively. The masses of the particles are taken unity. Note that the model is one dimensional, the $x$ variables denote positions in one chain, and the $y$ variables denote positions in the other chain. The particles are allowed to move only along the chain axis.

The ideal crystal is perfectly incommensurate and infinitely long. Because a computer cannot deal with such systems, in numerical calculations it has to be approximated. An approximant to the ideal crystal is made by considering a large unit cell that contains $p$ particles in the first chain and $q$ particles in the second chain, which is under the constraint of periodic boundary conditions. The infinite summations in the potential energy (1) are thus replaced by summations up to $p$ and $q$ particles. The ratio of $p$ and $q$ approximates an irrational number. This article uses neighboring numbers of the Fibonacci series for $p$ and $q$, the ratio $p / q=\alpha / \beta$ then approximates the golden mean $\tau=(1+\sqrt{5}) / 2$. The higher the neighboring numbers are, the better the approximation is. The behavior of the system for other ratios (no approximants of an incommensurate system) is studied as well. Systems with these different ratios are studied in Section III C.

The interchain potential $\Phi(r)$ was chosen to be a Lennard-Jones potential:

$$
\Phi(r)=2 k_{12}\left[\left(\frac{r^{2}+d^{2}}{\sigma^{2}}\right)^{-6}-\left(\frac{r^{2}+d^{2}}{\sigma^{2}}\right)^{-3}\right],
$$

where $k_{12}$ is the force constant of the interchain potential and $d$ the fixed distance between the two chains. The parameters $\sigma$ and $d$ equal 1 and 1.2, respectively in the numerical calculations. Figure 2 shows the Lennard-Jones potential. The well of the Lennard-Jones is at a smaller distance (at $r$ $=1.12$ ) than the distance $d$. Hence, only the attractive part beyond the minimum of the Lennard-Jones potential plays a role. The potential is cut off at a large distance, in these calculations it has a range of seven unit cells. A smooth steplike function at the endings prevents singularities in the derivatives of the potential energy. The Lennard-Jones potential introduces fairly long-range interactions, in contrast to the previously used Gaussian potential. That is, the tail of the Lennard-Jones-like interaction is longer than that of the

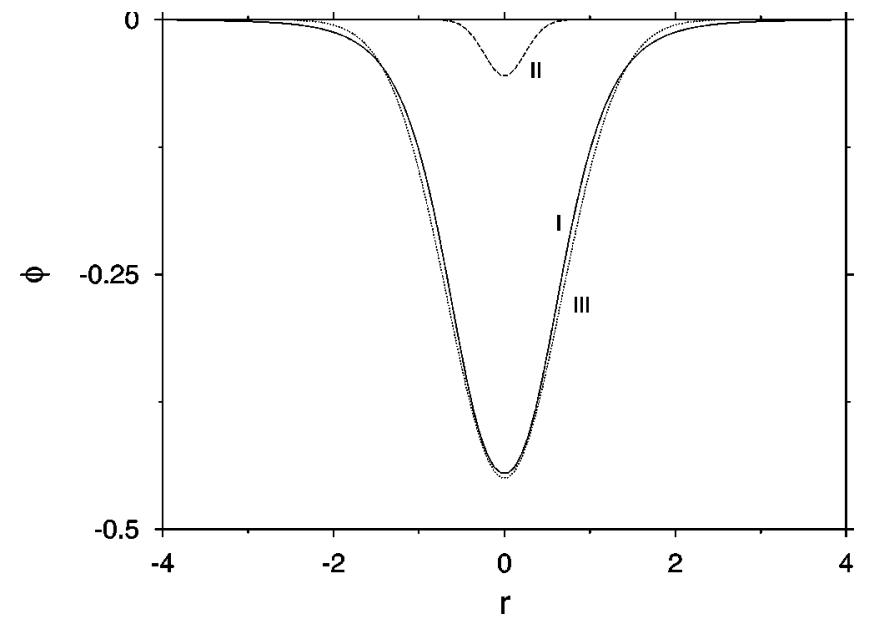

FIG. 2. The interchain potential. The solid line (I) indicates the Lennard-Jones-like interaction, the dashed line (II) the Gaussian interaction used in previous ${ }^{8}$ work and the dotted line (III) the Gaussian interaction which is as strong as the Lennard-Jones-like interaction. The variable $r$ is the distance between two particles situated in different chains.

Gaussian interaction, therefore the Lennard-Jones is called long range and the Gaussian short range.

\section{PHASE DIAGRAM}

\section{A. Modulation functions}

Ground states of the double chain model can be found by minimizing the potential energy (1). A quasi-Newton (the Broyden-Fletcher-Goldfarb-Shanno algorithm ${ }^{10}$ ) method was used to derive the equilibrium configuration in the numerical simulations. The equilibrium configurations of the chains in absence of the interchain interaction were used as a starting point for the minimalization routine and the convergence tolerance was taken to be $1.0^{*} 10^{-15}$.

If the interchain potential is weak compared to the intrachain interactions, a continuum of ground states is found. This implies that the potential energy does not change when the chains are moved with respect to each other. However, when the interchain potential gets stronger, a transition is found. Above the threshold value, neighboring ground states are separated by Peierls Nabarro barriers and energy is needed to go from one ground state to another.

The ground states can be studied by introducing modulation functions in the same way as they are introduced in, for instance, the Frenkel-Kontorova model. ${ }^{11}$ These modulation functions can be defined as follows:

$$
\left.\begin{array}{c}
x_{i}=\alpha i+f(i) \\
y_{j}=\beta j+g(j)
\end{array}\right\},
$$

where $x_{i}$ and $y_{j}$ are the positions of the particles in the ground state situated in the first and second chain, respectively, $\alpha i$ and $\beta j$ are the equilibrium (initial) positions of the particles without interchain interaction, and $f(i)$ and $g(j)$ are the displacements of the particles with respect to their initial positions. The modulation function of the first chain depends 

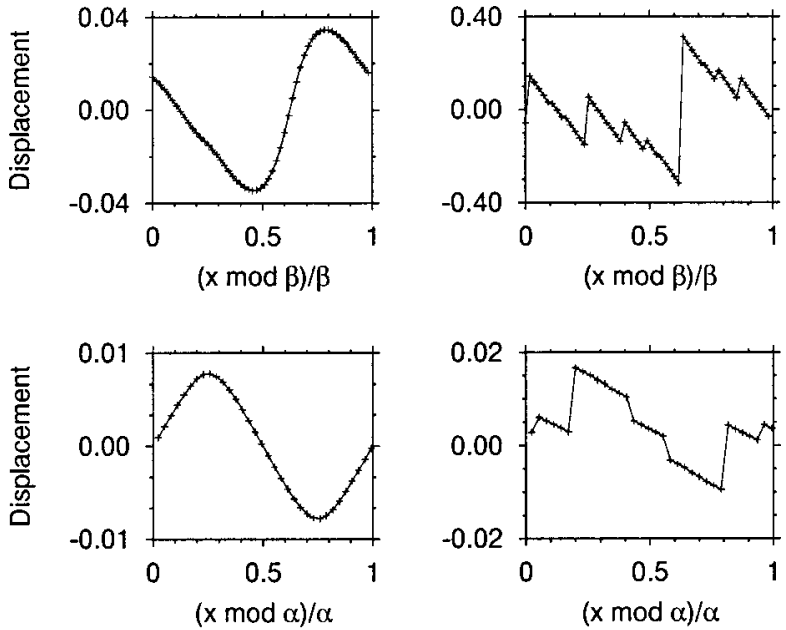

FIG. 3. The modulation functions. The upper two plots show the modulation function of the first chain, which contains 55 particles, the lower two plots that of the second chain, which contains 34 particles. The plots on the left are for a system in the analytic phase, on the right in the nonanalytic phase. The model parameters are $k_{2}=2, k_{12}=1, \alpha=1$, and $k_{1}=2$ for the former and $k_{1}=0.5$ for the latter.

on the configuration of the second chain and vice versa. Therefore, the periodicity of the modulation of one chain equals the periodicity of the other chain. Hence, $f(i)$ can be mapped on one period $\beta$ and $g(j)$ on one period $\alpha$. Equations (3) result in:

$$
\left.\begin{array}{l}
f(i \alpha \bmod \beta)=x_{i}-\alpha i \\
g(j \beta \bmod \alpha)=y_{j}-\beta j
\end{array}\right\} .
$$

If the ratio $\alpha / \beta$ is incommensurate, the points in the domain of the modulation functions are dense within one period. For a commensurate ratio, only discrete points are found.

Calculations of the modulation functions, for different values of the force constants, show analytic and nonanalytic regions. If the interchain potential is weak in comparison with the intrachain potentials, the modulation functions of both chains are analytic. That is, a smooth periodic function fits the points of the modulation functions. For strong interchain coupling compared to the intrachain coupling, the analyticity of the modulation functions is broken and gaps are introduced. Note that the analyticity breaks simultaneously for the two chains. In Fig. 3 our results are shown. The critical value of the interchain coupling is the same value that was mentioned before at which the continuity of the set of ground states was broken. This breaking of analyticity is a phase transition from an analytic to a nonanalytic phase. In subsection III $\mathrm{C}$ the opening of gaps in the modulation functions will be discussed in more detail.

\section{B. Phason gap}

The dynamical properties of the double chain model are derived from the eigenvalues and eigenvectors of the dynamical matrix. The elements of this matrix are defined as follows:
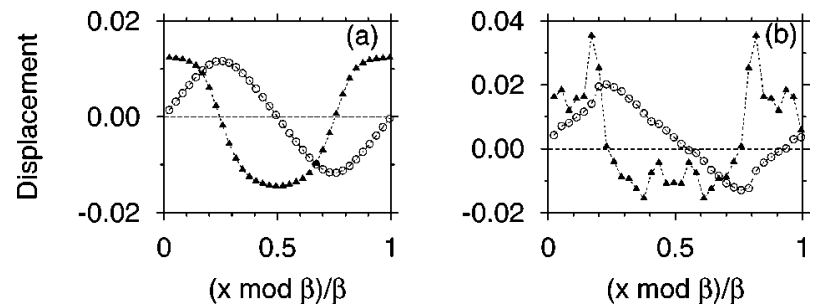

FIG. 4. The circles correspond to the modulation function, the triangles to the eigenvector of the phason mode. (a) The system is in the analytic regime. (b) The system is in the nonanalytic region, but near the transition line. The parameters of the system are $p$ $=55, q=34, k_{12}=1, \alpha=1$, and $d=1.2$, and $k_{1}=k_{2}=2$ in the case of (a) and $k_{1}=1.2$, and $k_{2}=2.0$ for the system of (b).

$$
D_{i j}^{z z^{\prime}}(k)=\left.\sum_{l} \frac{\partial^{2} E}{\partial z_{i} \partial z_{j}^{\prime}} \exp [-i k R(l)]\right|_{\mathrm{eq}},
$$

where $z$ and $z^{\prime}$ stand for $x$ or $y, E$ is the potential energy (1), $k$ is a wave vector, and $R(l)$ is the equilibrium position of the $l$ th unit cell. ${ }^{12}$ The frequencies of the phonon excitations in the crystal are given by the square roots of the eigenvalues of the dynamical matrix. The corresponding displacements are given by:

$$
u_{s}^{i}(R(l))=\operatorname{Re}\left\{\epsilon_{s}^{i}(k) \exp [i k R(l)]\right\},
$$

where $s$ denotes the mode, $i$ the number of the particle in the primitive cell, and $\epsilon_{s}$ the eigenvector corresponding to mode $s$. The dynamical matrix is infinitely dimensional if the crystal is truly incommensurate. However, because the crystal is approximated by a large unit cell to which periodic boundary conditions are applied, the Bloch theorem may be used to decompose $D$ into a family of finite dynamical matrices. If the system is in the analytic region, the eigenvector corresponding to the phason mode is the derivative of the modulation function. ${ }^{13}$ Figure 4(a) shows the modulation function of the second chain and the eigenvector of the phason, where the latter is clearly the derivative of the first. When the system is just outside the analytic region, the phason can also be seen as the derivative of the modulation function. That is, at gaps in the modulation function also the phason eigenvector shows discontinuities (though they are finite) and the slopes of the continuous parts of the modulation correspond to the phason eigenvector, as Fig. 4(b) shows.

The dispersion relations are constructed using the eigenvalues for different values of the wave vector $k$. Figure 5 shows the dispersion relations for two specific systems, in Fig. 5(a) the system is in the analytic region, in Fig. 5(b) in the nonanalytic region. The system in the analytic region has two modes at zero wave vector with zero frequency. One corresponds to the acoustic (Goldstone) mode, the other to the phason or sliding mode. The first mode is a uniform displacement of the particles in the unit cell. The second corresponds to a mode where the two chains move in opposite directions. As the interchain potential increases, the degeneracy is lifted which introduces the phason gap. This occurs at the same point where the analyticity of the modulation functions breaks. Figure 5(b) shows such a pha- 

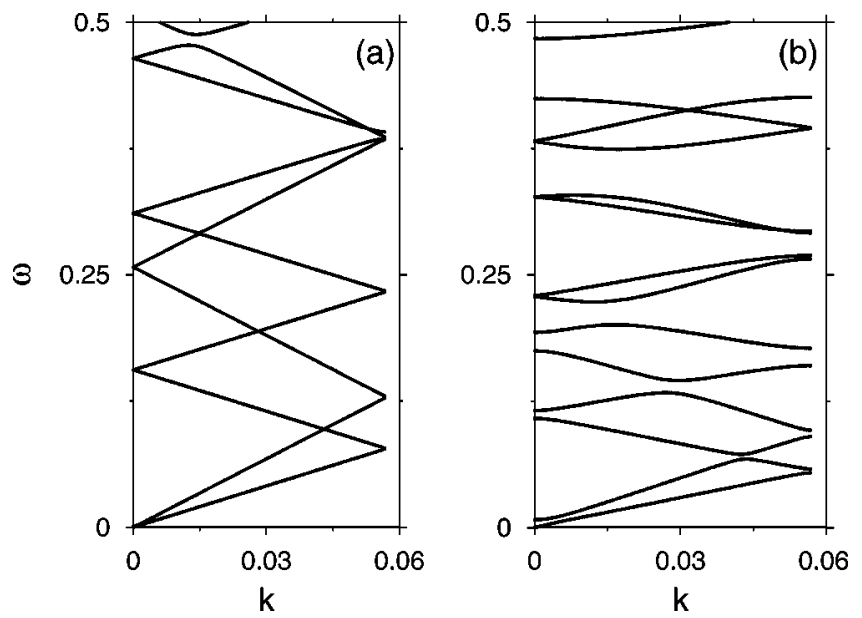

FIG. 5. Dispersion relations. This figure shows part of the dispersion relations. The parameters of the system are $p=55, q=34$, $k_{12}=1, \alpha=1$, and $d=1.2$, and $k_{1}=k_{2}=2$ in the case of (a) and $k_{1}=k_{2}=1.2$ for the system of (b). (a) At $\Gamma$ there are two modes which have (almost) zero frequency. One is the acoustic mode, the other is the phason mode. Due to the weak interaction some bandcrossings open. The model is in the analytic phase. (b) Now the degeneracy disappeared and the phason gap opened. The system is here in the nonanalytic phase.

son gap. This gap is defined as the difference between the frequencies of the phason mode and the acoustic mode. The phason mode is defined as the mode showing the largest displacement of the chains in opposite directions and is not always the second mode in the dispersion. Crossing the transition line from the nonanalytic to the analytic phase the gap closes rapidly. Figure 6 shows the closing of the gap if $k_{2}$ and $k_{12}$ are kept constant and $k_{1}$ is varied for two approximants. As can be seen the gap falls off rather quickly, like

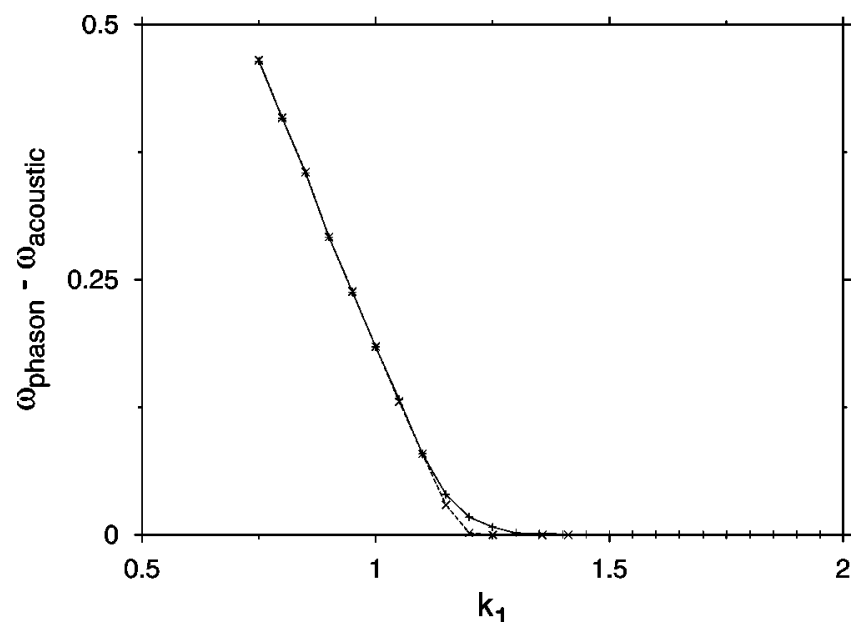

FIG. 6. Closing of the phason gap. Only one of the force constants $\left(k_{1}\right)$ changes, the other two are fixed. As can be seen, the gap closes quickly. The solid line describes the closing of the gap for a system with $p=55$ and $q=34$, the dashed line for a system with $p=144$ and $q=89$. Both approximants almost coincide entirely. The other system parameters are $k_{12}=1, \alpha=1, d=1.2$, and $k_{2}$ $=2$.

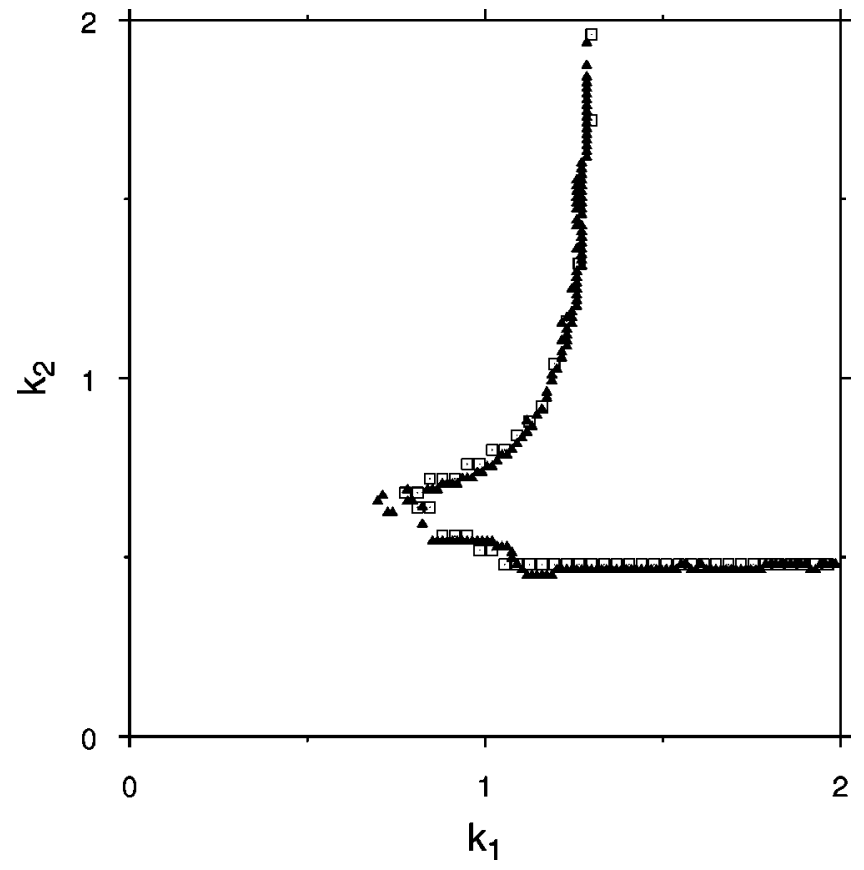

FIG. 7. The phase diagram, obtained in two different manners for the Lennard-Jones-like interchain potential. The squares indicate the breaking of analyticity of the modulation functions of the two chains, the triangles the disappearance of the phason gap. The system parameters are $p=55, q=34, k_{12}=1, \alpha=1$, and $d=1.2$.

$\exp \left(-k_{1}^{3}\right)$. The two lines coincide everywhere, except close to the $k_{1}$ axis, so the lower approximant already gives a good result.

\section{The phase diagram}

The previous two subsections showed that for weak interchain interaction compared to the intrachain interactions, the modulation functions are both analytic and no phason gap exists. If the interchain coupling exceeds a threshold, the analyticity of the modulation functions breaks and the phason gap opens, both at the same time. At this threshold a phase transition from an analytic to a nonanalytic regime takes place. Whether the system is in the analytic or nonanalytic region depends on the force constants, although one of them, e.g., $k_{12}$, can be scaled away. The actual shape of the transition line in the phase diagram depends on the ratios $k_{1} / k_{12}$ and $k_{2} / k_{12}$.

Figure 7 shows the phase diagram of an approximant which has 55 particles in one chain and 34 in the other chain. The diagram was obtained in two ways, using the analyticity breaking of the modulation functions and the opening of the phason gap. As the figure shows, the two lines coincide very well. Note that the transition line is asymmetric. The value of $k_{2}$ at which the transition takes place for high values $k_{1}$ is not the same value of $k_{1}$ for high values of $k_{2}$. Another result is the nonconvex part in the transition line. If in this region $k_{1}$ is fixed, for increasing $k_{2}$ the system first passes the transition line from the nonanalytic to the analytic region, later on it passes the line again and enters the nonanalytic region.

In the numerical calculations, a criterion has to be chosen whether the transition line is crossed. The approximants are 


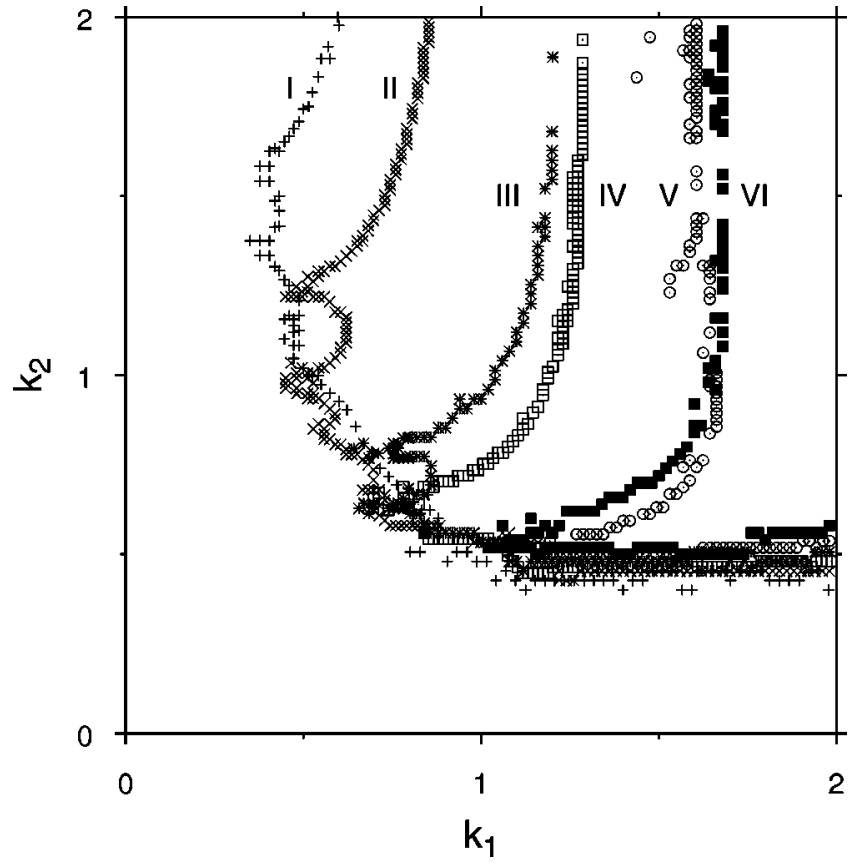

FIG. 8. Phase diagrams for different ratios of the periods of the two chains. For all cases, the number of particles in chain 2 is 34 . The number of particles in chain 1 is 47 (I), 49 (II), 53 (III), 55 (IV), $59(\mathrm{~V})$, and 57 (VI). The corresponding ratios are 1.38, 1.44, $1.56,1.62,1.74$, and 1.68 , respectively. There is no line in case of 51 particles in the first chain (ratio is $3 / 2$ ), because this is a truly commensurate case.

not truly incommensurate crystals, in fact they are commensurate. As a consequence, there will always be a finite number of points in the domains of the modulation functions, i.e., they are not densely spread. Nonetheless, a nonanalytic and an analytic regime can be distinguished. In the analytic region, the points of the modulation functions can be fitted by a smooth periodic function, whereas, in the nonanalytic region clearly gaps appear (see Fig. 3).

A similar argument holds for the phason gap. If a crystal is truly commensurate, it will always possess a phason gap. However, in case of the approximants, there is a region with a large gap in respect to another region which contains a tiny but finite gap. At the transition line, which divides those two regions, the phason gap closes rapidly as Fig. 6 shows. If the phason gap is less then 0.004 , the system is considered to be in the analytic region.

The existence of the phason mode was used to determine also the phase diagrams of systems with a different ratio than the golden mean. The results are depicted in Fig. 8. The transition line becomes more asymmetric and the nonconvex region increases for increasing number of particles in the first chain, where the number in the other chain is kept fixed. The diagram for the approximant with $p / q \approx \tau$ is not a special case, i.e., it is not an extremum beyond which no other transition lines for other ratios are found. Note that the transition line for the case where the system contains 51 particles in the first and 34 particles in the second chain lacks. In this case the ratio of $p$ and $q$ is commensurate with small denominator

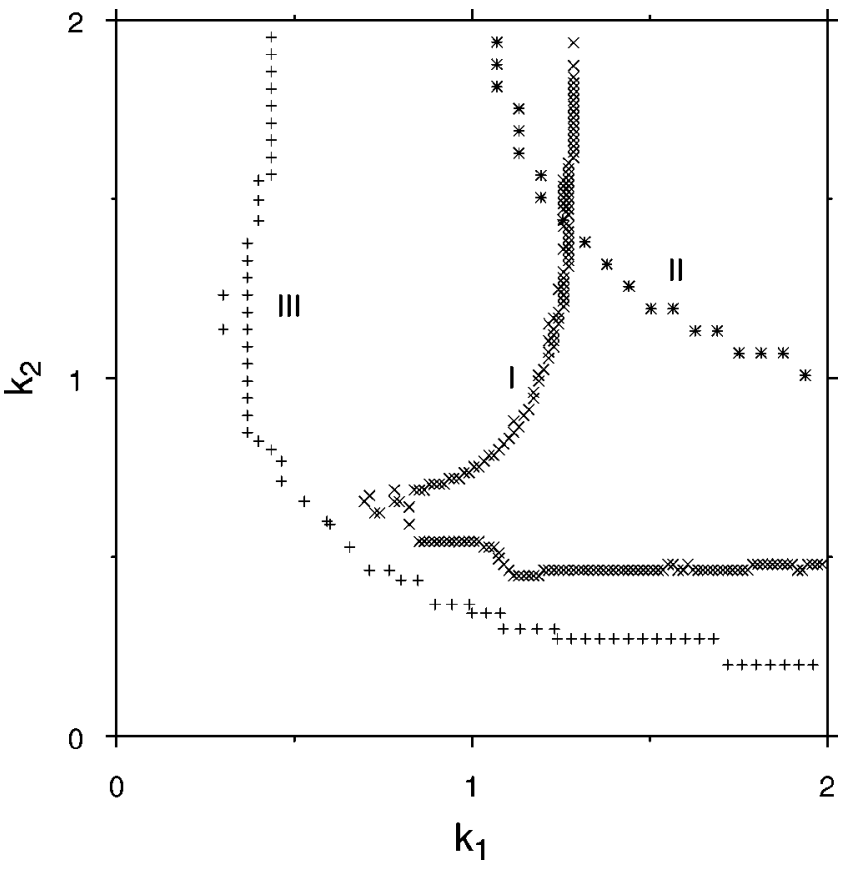

FIG. 9. Phase diagrams with different interchain potentials shown in Fig. 2. Line I indicates the phase diagram of the model with the Lennard-Jones potential, line II that of the short-range Gaussian potential, and line III that of the deeper Gaussian potential. The system parameters are $p=55, q=34, k_{12}=1, \alpha=1$, and $d=1.2$.

$(3 / 2)$ and therefore the system never shows a gap as small as the other approximants. Thus the actual ratio of $p / q$ deserves special attention.

As already mentioned, in previous work $^{8}$ a Gaussian potential of the form:

$$
\Phi(r)=-\exp \left(-r^{2}\right)
$$

was used instead of the Lennard-Jones potential. Figure 9 shows the results for the Gaussian and the Lennard-Jones potential for an approximant of the incommensurate crystal. The transition line for the Gaussian is symmetric and convex. As Fig. 2 shows, the two potentials differ in range and in magnitude. Therefore another Gaussian was taken as to make a better comparison. The well of the Gaussian was made as deep as that of the Lennard-Jones potential. Note that the ranges are still very different, the Gaussian goes to zero exponentially, whereas the Lennard-Jones potential goes as $r^{-6}$. Figure 9 shows also the phase diagram for this Gaussian potential. As can be seen, the transition line is symmetric and convex, just as in the case of the other Gaussian.

In case of the Lennard-Jones-like potential an asymmetric transition line was found. This in contrast to the transition line found using the Gaussian potentials. The asymmetry was found when the ratio of the numbers of particles in the chains does not approach an integer number. The impact of this irregularity in the input parameters becomes apparent for the long range interchain interaction. The sensitivity of the Gaussian potential is negligible for this long-range effect. Note that in the model used to examine friction ${ }^{9}$ reentrant behavior was also found. In this model a Gaussian interchain 

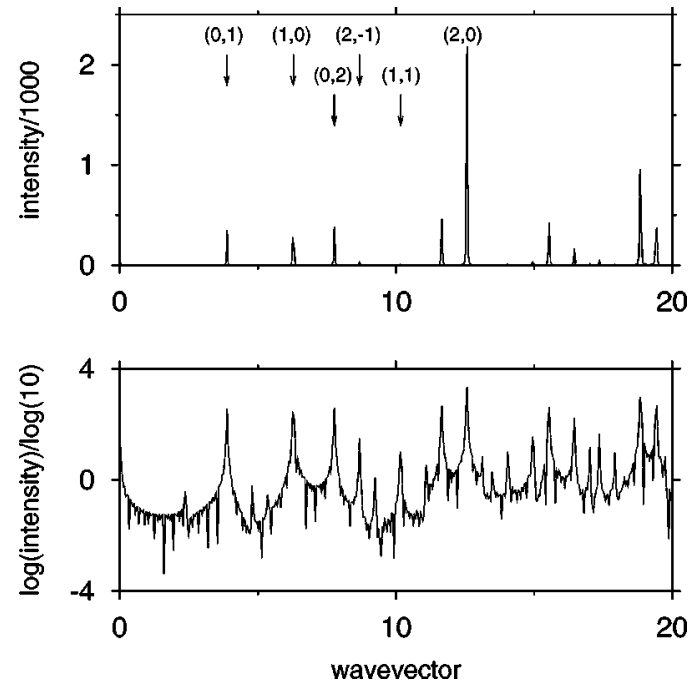

FIG. 10. The geometrical structure factor. The $x$ axis is the difference in incoming and outgoing wave vectors, the $y$ axis is the calculated intensity. The lower plot shows the logarithm of the upper plot. The labels $(n, m)$ correspond to those used in Table I. The model is in the analytic regime, its parameters being $p=55, q$ $=34, k_{1}=k_{2}=2, k_{12}=1, \alpha=1$, and $d=1.2$.

potential was used in a two-chain structure where one chain was attached to a bulk. This model shows a different structure, i.e., one chain is attached to a bulk whereas the other chain is free, which probably causes the reentrant behavior in this system. A further elaboration on these asymmetries is out of the scope of this article that concentrates on differences between long- and short-range interchain interactions.

\section{OBSERVING THE PHASON MODE}

Attempts have been made to observe the phason or sliding mode in incommensurate compounds. There is a claim ${ }^{2}$ that this mode is measured in alkane/urea, but this result is disputed by others. ${ }^{3}$ So, the question now is, what are the conditions to observe a sliding mode. When the crystal is in the analytic part or just near the transition line in the nonanalytic part of the phase diagram, the phason branch will be the second branch. By x-ray diffraction the main Bragg and satellite peaks can be determined. At these peaks, the phason mode can be measured if it exists, as here the intensities of the phonon modes will be large. Inelastic neutron scattering, for instance, might reveal the phason mode.

Using $\mathrm{x}$-ray or neutron scattering, main Bragg peaks will be found at $K_{\alpha}=2 n \pi / \alpha$ and $K_{\beta}=2 m \pi / \beta$, with $n$ and $m$ integers. The former are just the Bragg reflections due the first chain, the latter are due to the second chain. Besides these main reflections there also exist satellite peaks. These can be found at $K_{\alpha}+K_{\beta}=2 n \pi / \alpha+2 m \pi / \beta$, where both $n$ and $m$ are nonzero. The labeling of the peaks will be $(n, m)$, where $n$ stands for contributions of the host and $m$ of the guest. Both the main and satellite reflections can be given in terms of the Bragg reflections of the large unit cell $K$ $=2 l \pi / \alpha p$, where $l$ is an integer. The relative intensities of the reflections can be calculated using the geometrical structure factor:
TABLE I. Some intensities of the acoustic and phason mode. The subscript $p$ denotes the phason, $a$ the acoustic mode. The velocity of the phason mode equals 2.246, of the acoustic mode 1.363. Four main Bragg peaks and two satellite peaks and their neighborhoods are examined. The boundary of the first Brillouin zone is at 0.057 . The indices of the peaks are $(0,1),(0,2),(1,0),(2,0),(2,-1)$, and $(1,1)$, respectively. The system is in the analytic region of the phase diagram, its parameters being $p=55, q=34, k_{1}=k_{2}=2$, $k_{12}=1, \alpha=1$, and $d=1.2$.

\begin{tabular}{|c|c|c|c|c|c|}
\hline$k$ & $\begin{array}{c}\omega_{p} \\
* 10^{-2}\end{array}$ & $\begin{array}{c}\omega_{a} \\
* 10^{-2}\end{array}$ & $\begin{array}{l}S\left(\omega_{p}\right) \\
* 10^{2}\end{array}$ & $\begin{array}{l}S\left(\omega_{a}\right) \\
* 10^{2}\end{array}$ & $\frac{S(\omega)_{p}}{S(\omega)_{a}}$ \\
\hline \multicolumn{6}{|l|}{$(0,1)$} \\
\hline 3.89 & 2.3 & 1.4 & 31.75 & 0.67 & 47.05 \\
\hline 3.90 & 4.5 & 2.7 & 23.13 & 0.43 & 54.17 \\
\hline $\begin{array}{l}3.91 \\
(0,2)\end{array}$ & 6.8 & 4.1 & 12.89 & 0.21 & 62.64 \\
\hline 7.78 & 2.3 & 1.4 & 23.71 & 0.48 & 49.72 \\
\hline 7.79 & 4.5 & 2.7 & 17.30 & 0.30 & 57.80 \\
\hline $\begin{array}{l}7.80 \\
(1,0)\end{array}$ & 6.8 & 4.1 & 9.66 & 0.15 & 63.16 \\
\hline 6.29 & 2.3 & 1.4 & 0.67 & 40.36 & 0.02 \\
\hline 6.30 & 4.5 & 2.7 & 0.41 & 29.51 & 0.01 \\
\hline $\begin{array}{l}6.31 \\
(2,0)\end{array}$ & 6.8 & 4.1 & 0.19 & 16.51 & 0.01 \\
\hline 12.58 & 2.3 & 1.4 & 0.82 & 39.54 & 0.02 \\
\hline 12.59 & 4.5 & 2.7 & 0.67 & 28.81 & 0.02 \\
\hline $\begin{array}{l}12.60 \\
(2,-1)\end{array}$ & 6.8 & 4.1 & 0.43 & 16.06 & 0.03 \\
\hline 8.69 & 2.3 & 1.4 & 0.40 & 1.18 & 0.34 \\
\hline 8.70 & 4.5 & 2.7 & 0.31 & 0.77 & 0.40 \\
\hline $\begin{array}{l}8.71 \\
(1,1)\end{array}$ & 6.8 & 4.1 & 0.19 & 0.38 & 0.51 \\
\hline 10.18 & 2.3 & 1.4 & 0.17 & 0.32 & 0.54 \\
\hline 10.19 & 4.5 & 2.7 & 0.18 & 0.20 & 0.86 \\
\hline 10.20 & 6.8 & 4.1 & 0.13 & 0.10 & 1.35 \\
\hline
\end{tabular}

$$
I(k) \propto|S(k)|^{2}=\sum_{i}^{n} \sum_{j}^{n} \exp \left[i k\left(z_{i}-z_{j}\right)\right],
$$

where $z$ stands for $x$ or $y$, the positions of all the particles in the unit cell and $k$ is the difference between incoming and outgoing wave vector. Figure 10 shows the intensities for an approximant in the analytic phase. The lower graph shows the logarithm of the intensity. Clearly, for higher values of the wave vector, the intrinsic computational inaccuracies increase, as is to be expected. This figure shows also that the peaks are dense. This is because the approximant has a very small Brillouin zone, and therefore the Bragg reflections of the large unit cell given by $K$ above, will all show some intensity, though many of these peaks will be very small. More satellite peaks can be discovered in the logarithmic figure.

The response function which gives information about the phonon spectrum of a crystal, is the dynamic structure factor. 
For a fixed wave vector $k$, the intensities in a neutronscattering experiment can be calculated using:

$$
S(k, \omega)=\sum_{s}\left|\sum_{j} \epsilon_{s}^{j} \exp \left[i k z_{j}\right]\right|^{2} \delta(\omega-w(s)),
$$

where $\epsilon_{s}^{j}$ are the eigenvectors of the dynamical matrix (which are complex outside $\Gamma$ ), $z_{j}$ is the position of the particle, $k$ is the wave vector, $\omega$ is the frequency of the wave and $w(s)$ are the square roots of the eigenvalues of the dynamical matrix.

Table I shows calculations around Bragg and satellite peaks for a specific choice of parameters. If the static structure factor shows a relative high intensity at a Bragg or satellite peak, the scattering of neutrons by phonons can be measured better near these peaks than at reflections with much lower intensity of the static structure factor. The phason mode appears both near main and satellite reflections. However, the relative intensity of the phonon modes at the satellite peaks is less than at the main peaks, as is to be expected from the relative intensities calculated by the static structure factor (see Fig. 10). Just next to the main $(0,1)$ reflection (first main Bragg peak of the guest lattice), the phason mode is much stronger than the acoustic mode. Whereas next to the main $(1,0)$ peak the acoustic mode has more intensity than the phason mode as is also the case for $(2,0)$. Near the calculated reflections the phason mode has more intensity than the acoustic mode near main peaks of the second chain. Near the satellite peaks, the phason mode has half the intensity of the acoustic mode. The velocity of the phason mode equals 2.246 and of the acoustic mode 1.363. If there is no coupling between the chains, the velocities are 2.291 and 1.410, respectively. When the wave vector increases, the intensities of the modes decrease. The Brillouin zone contains many branches. These branches are actually the branches of the dispersion relations of the two separate chains folded into the Brillouin zone of the unit cell. It turned out that halfway the Brillouin zone the lower folded branch decreases its intensity whereas the next folded branch (the two folded branches actually belong to the same chain) increases in intensity. Thus, the intensity is "taken over" half-way through the Brillouin zone by a higher mode.

\section{CONCLUSIONS}

Both the modulation functions and the existence of the phason gap lead to a phase diagram. At the transition line the analyticity of the modulation functions break and the phason gap appears. The transition line depends on the ratio of the force constants $k_{1} / k_{12}$ and $k_{2} / k_{12}$.

In the case of an approximant of an incommensurate crystal, the transition line is asymmetric and has a nonconvex part. The transition line of the approximant with $p / q \approx \tau$ is not an extremum, beyond which no other lines exist. For commensurate systems with a small denominator, such as the one with 51 particles in one chain and 34 in the other, the gap is always large compared to the gap of the approximant in the analytic region. As a consequence, no transition was found in this case.

Performing the same calculations for an approximant using a Gaussian interchain potential of depth comparable to that of the Lennard-Jones interaction, the transition line is more symmetric and does not contain a nonconvex region. Therefore, the asymmetry and the nonconvex shape are due to the long range of the Lennard-Jones-like potential. The range of the latter comprises seven unit cells, that of the Gaussian potentials is much smaller.

Just outside the main Bragg reflections of the second chain, the intensity of the phason mode is relatively larger than the intensity of the acoustic mode. The group velocity of the phason mode is larger than of the acoustic mode, so the second branch is the phason branch.

In future work, the one dimensional model will be extended to higher dimensions. Incorporating the actual values of the three force constants, the distance between the chains and the periods within one chain, makes it possible to make a better comparison with experimental work.

\section{ACKNOWLEDGMENTS}

This work was supported by Stichting Fundamenteel Onderzoek der Materie with financial support from the Nederlandse Organisatie voor Wetenschappelijk Onderzoek.
${ }^{1}$ D. Schmicker, S. van Smaalen, C. Haas, and K.D.M. Harris, Phys. Rev. B 49, 11572 (1994).

${ }^{2}$ D. Schmikker, S. van Smaalen, J.L. de Boer, C. Haas, and K.D.M. Harris, Phys. Rev. Lett. 74, 734 (1995).

${ }^{3}$ J. Ollivier, C. Ecolivet, S. Beaufils, and T. Breczewski, Europhys. Lett. 43, 546 (1998)

${ }^{4}$ J. Frenkel and T. Kontorova, Phys. Z. Sowjetunion 13, 1 (1938).

${ }^{5}$ F.C. Frank and J.H. Van der Merwe, Proc. R. Soc. London, Ser. A 198, 205 (1949).

${ }^{6}$ G. Theodorou and T.M. Rice, Phys. Rev. B 18, 2849 (1978).

${ }^{7}$ T. Ishii, J. Phys. Soc. Jpn. 52, 168 (1983).
${ }^{8}$ O. Radulescu and T. Janssen, Phys. Rev. B 60, 12737 (1999).

${ }^{9}$ T. Kawaguchi and H. Matsukawa, Phys. Rev. B 58, 15866 (1998).

${ }^{10}$ W. H. Press, S. A. Teukolsky, W. T. Vettering, and B. P. Flannery, Numerical Recipes in C, The Art of Scientific Computing, 2nd ed. (Cambridge University Press, Cambridge, 1996).

${ }^{11}$ L.M. Floria and J.J. Mazo, Adv. Phys. 45, 505 (1998).

${ }^{12}$ A.A. Maradudin, E.W. Montroll, and G.H. Weiss, Solid State Physics (Academic Press, New York, 1963).

${ }^{13}$ T. Janssen, Ferroelectrics 236, 157 (2000). 